\title{
Lab-on-chip with microalgal based biosensor for water assessment
}

\author{
F. Sekli Belaidi ${ }^{1,2}$, A. Tsopela ${ }^{1,2}$, L. Salvagnac ${ }^{1,2}$, V. Ventalon ${ }^{1,2}$, E. Bedel-Pereira ${ }^{1,2}$, V. Bardinal ${ }^{1,2}$, I. \\ Séguy ${ }^{1,2}$, P. Temple-Boyer ${ }^{1,2}$, P. Juneau ${ }^{3}$, R. Izquierdo ${ }^{3}$, J. Launay ${ }^{1,2}$
}

\begin{abstract}
The integration of optical source with microfluidics leads to promising low-cost, rapid and sensitive lab-on-a-chip analytical systems. This work deals with the optimization of the electrochemical sensors and the dedicated organic light-emitting diode (OLED) for the monitoring of herbicides though algal photosynthetic activities disturbances. . Various blue OLED are considered in order to achieve highest sensitivity in $\mathrm{O}_{2}$ electrochemical detection during micro-algae photosynthesis.
\end{abstract}

\section{INTRODUCTION}

Aquatic ecosystems are very sensitive to global climatic changes and other human impacts [1]. In the last decades, agricultural and urbanization expansion have induced increasing quantities of pollutant contaminations. It is therefore challenging to develop disposable systems for rapid and in situ analysis to assay toxicants traces in water. Our lab-on-chip structure consists in electrochemical sensors fully integrated on a microfluidic platform, fabricated on a glass substrate. Pollution level is estimated by electrochemical monitoring of oxygen during algae (Chlamydomonas Reinhardtii) photosynthesis in presence of different herbicide concentrations. In previous studies, we demonstrated that blue OLED based integrated system shows higher detection characteristics than those using external white light source [2]. The appeal of OLED as excitation sources in such a detection platform consists in their integration capacity and low fabrication costs. In this paper we propose to analyze algae photosynthesis using various wavelengths and power selective blue organic light-emitting diodes as stimulation light sources.

\section{LAB-ON-CHIP DESCRIPTION}

\section{A. Electrochemical sensor}

The integrated three electrode electrochemical microcells (working, reference, and counter electrodes) are developed through photolithography patterning, followed by physical vapor deposition (PVD) process of titanium/platinum (Ti/Pt) films. $\mathrm{A} \mathrm{Si}_{3} \mathrm{~N}_{4}$ layer deposited by inductively coupled plasma chemical vapor deposition ICP-CVD is used as long-term passivation. Microfluidic platform is structured by patterning SU8 photoresist. Platinum electrodes are directly used as counter electrodes, while working electrodes are functionalized by platinum black deposition to enhance the

\footnotetext{
*Research supported by ABC Foundation.

(1) CNRS, LAAS, 7 avenue du colonel Roche, F-31400 Toulouse, France

(2) Université de Toulouse, UPS, LAAS, F-31400 Toulouse, France. E.mail : fsekli@laas.fr

(3) Université du Québec à Montréal, 201 Président Kennedy, Montréal, Canada.
}

electrochemical detection of $\mathrm{O}_{2}$. To create the integrated reference electrodes, silver layers are electroplated directly on platinum surface and then oxidized to obtain $\mathrm{Ag} / \mathrm{AgCl}$. This method gives stable and durable integrated reference electrodes.

\section{B. Excitation source}

In order to demonstrate the versatility of OLED technology and its impact on algae oxygen production, we plan to (i) place OLED on the top of the detection chamber to validate this device as excitation source and (ii) fabricate three different OLEDs to test their efficiency. Key technical issue for developing the suitable exciting source is to obtain the appropriate light intensity and spectral overlap between OLED emission and algal absorption displayed in Fig.1. Table 1 summarizes the configuration of different blue OLED (devices A-C) fabricated by thermal evaporation on indium thin oxide (ITO) coated glass under high vacuum conditions.

TABLE I. STRUCTURE OF BLUE OLEDS

\begin{tabular}{|c|c|}
\hline Device & Organic thin film stack \\
\hline A & $\mathrm{NPB}(20 \mathrm{~nm}) / \mathrm{PCAN}(40 \mathrm{~nm}) / \mathrm{Alq}_{3}(20 \mathrm{~nm})$ \\
\hline $\mathrm{B}$ & $\mathrm{NPB}(80 \mathrm{~nm}) / \mathrm{Bphen}(30 \mathrm{~nm})$ \\
\hline $\mathrm{C}$ & HAT-CN $(10 \mathrm{~nm}) / \mathrm{NPB}(35 \mathrm{~nm}) / \mathrm{TAPC}(5 \mathrm{~nm}) / \mathrm{MADN}(20 \mathrm{~nm}) /$ \\
\hline
\end{tabular}

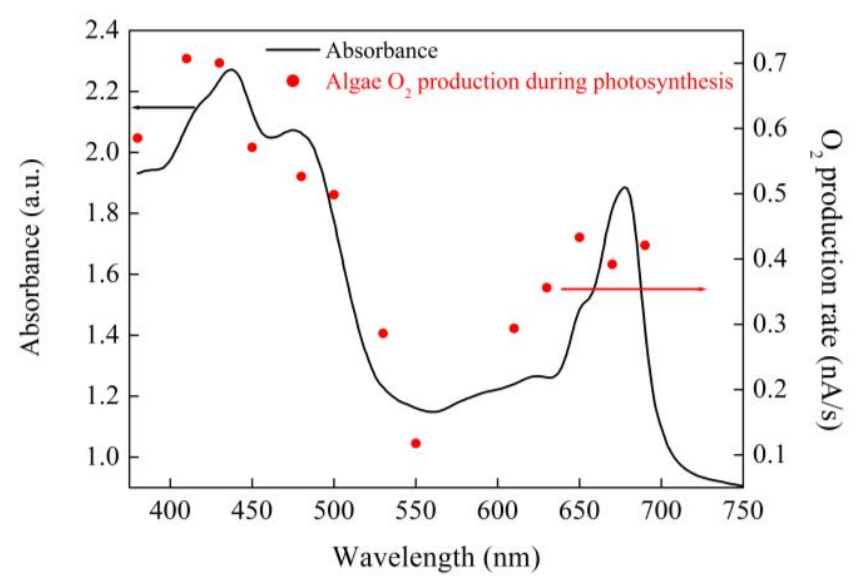

Figure 1. Chlamydomonas reinhardtii absorption spectra in High Salt Medium with the adjusted $\mathrm{pH}$ 6.8. and $\mathrm{O}_{2}$ production during photosynthesis at different excitation wavelengths. 


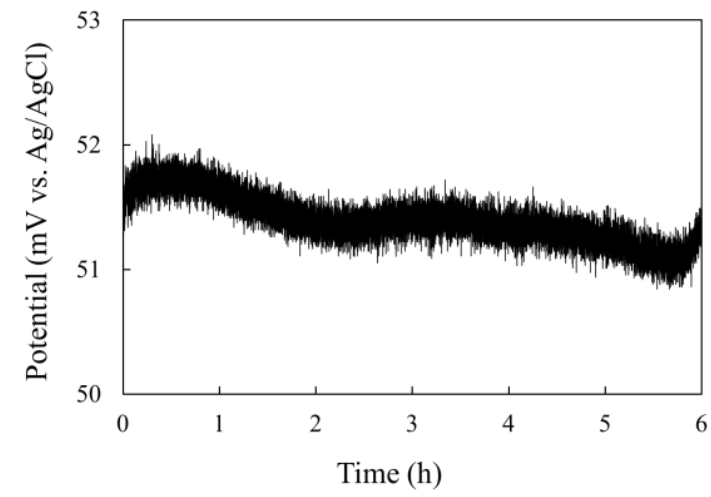

Figure 2. Open circuit potential of $\mathrm{Ag} / \mathrm{AgCl}$ reference microelectrode in $0.1 \mathrm{M}$ potassium chloride solution vs an $\mathrm{Ag} / \mathrm{AgCl}$ commercial reference electrode.

OLED are composed of HAT-CN as hole injection layer (HIL), NPB as hole transport layer (HTL), PCAN, NPB and MADN as emitting layers (respectively for devices A, B and C), $\mathrm{Alq}_{3}$ and Bphen as electron transport layers (ETL). Then a $100 \mathrm{~nm}$ aluminum cathode is deposited by thermal evaporation.

\section{RESULTS AND DISCUSSION}

\section{A. Reference microelectrode stability}

The identified critical limitation of integrated electrochemical sensors concerns the reference electrode stability. In this work, we choose a very simple electrode preparation by electrochemical Ag deposition thanks to a silver nitrate solution (constant current), followed by linear sweep voltammetry for the $\mathrm{Ag}$ surface chlorination in saturated potassium chloride solution. Ag layer thickness needs to be thick enough not to be completely chlorinated into $\mathrm{AgCl}$, but not too thick to avoid cracks in the obtained film. This approach yields to a $5 \mu \mathrm{m}$ stable and homogeneous $\mathrm{Ag} / \mathrm{AgCl}$ structure. In order to determine this reference electrode stability, the open circuit potential (OCP) versus a standard reference commercial electrode is monitored in $\mathrm{KCl}$ solution (Fig. 2). The OCP measurements can be done with different chloride concentrations, which allow verifying the potential dependence of the $\mathrm{Ag} / \mathrm{AgCl}$ electrode with the chloride ions concentration. In $0.1 \mathrm{M} \mathrm{KCl}$ solution we observe an offset of $51.7 \mathrm{mV}$, smaller than the value given by the Nernst equation $(60 \mathrm{mV})$ and a drift value of about $20 \mu \mathrm{V} / \mathrm{h}$. The potential derivations shown are slightly better than other reference microelectrodes reported in literatures [3].

\section{B. OLEDs algae stimulation and $\mathrm{O}_{2}$ monitoring}

Algae absorption spectrum (Fig. 1) presents two large absorption bands located in blue and red regions. First results obtained with Xenon excitation source, show that oxygen monitoring during algae photosynthesis measured with electrochemical sensors described in the first part of this paper exhibit higher sensitivity with blue excitation light (Fig. 1).

The aim of this work is to take advantage of OLED technology to select devices with the organic stack better fitted to further improve the sensor sensitivity.

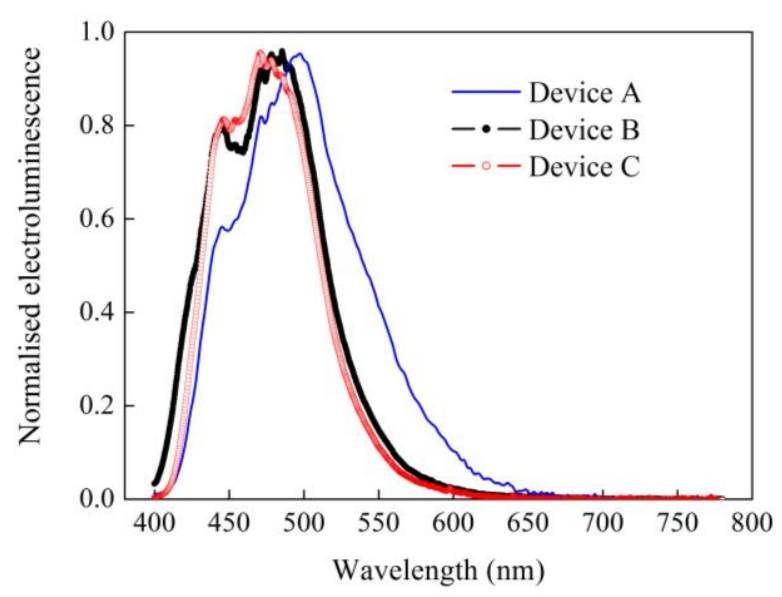

Figure 3. Normalised electroluminescence spectra (the spectra are normalised with respect to the maximum emission) of devices A, B and C.

For the three OLED configurations A, B and C reported in table 1 , blue fluorescence is observed above $8 \mathrm{~V}$. The figure 3 evidence that by replacing the $\mathrm{Alq}_{3}$ electron transport layer with Bphen thin films, two types of blue emitting OLED are obtained: sky-blue (device A) or deep blue (device B and C). In the third generation of OLEDs (device $\mathrm{C}$ ) organic layers have been added in component structure, in order to first manage hole injection and then confine exciton in emitting layer leading to higher devices efficiency and lifetime.

Studies are in progress to examine the role played whether by OLED emission spectra (device A versus devices B and $\mathrm{C}$ ), light intensity, and temperature management (device B versus device $\mathrm{C}$ ) in Chlamydomonas Reinhardtii photosynthesis in such lab-on-chip platforms.

\section{ACKNOWLEDGMENT}

The authors would like to thank the french "Agence Nationale de la Recherche" (ANR, project Dolfin, no. anr13-js03-0005-01) and the Fonds France-Canada pour la recherche (FFCR) for financing the project. Furthermore, microfabrication procedure was partly supported by the French Renatech network.

\section{REFERENCES}

[1] P.K .Bharti, "Anthropogenic Activities and Global Climate Change In: Climate Change and Agriculture", Discovery Publishing House, New Delhi, India, 2012, pp. 1-22.

[2] A. Tsopela, A.Laborde, L. Salvagnac, V. Ventalon, E. Bedel-Pereira, I. Seguy, P. Temple-Boyer, P. Juneau, R. Izquierdo, J. Launay, "Development of a lab-on-chip electrochemical biosensors for water quality analysis based on microalgal photosynthesis, Biosensors and bioelectronics 79, 2016, pp. 568-573

[3] I. Y. Huang, R. S. Huang, "Fabrication and characterization of a new planar solid-state reference electrode for ISFET sensors", Thin solid films 406, 2002, pp. 255-261 